\title{
How generation affects source memory
}

\author{
KINDIYA D. GEGHMAN and KRISTI S. MULTHAUP \\ Davidson College, Davidson, North Carolina
}

\begin{abstract}
Generation effects (better memory for self-produced items than for provided items) typically occur in item memory. Jurica and Shimamura (1999) reported a negative generation effect in source memory, but their procedure did not test participants on the items they had generated. In Experiment 1, participants answered questions and read statements made by a face on a computer screen. The target word was unscrambled, or letters were filled in. Generation effects were found for target recall and source recognition (which person did which task). Experiment 2 extended these findings to a condition in which the external sources were two different faces. Generation had a positive effect on source memory, supporting an overlap in the underlying mechanisms of item and source memory.
\end{abstract}

The term source memory refers to identifying where information originated. Johnson, Hashtroudi, and Lindsay (1993) argued that source memory can involve discriminating between external sources (e.g., two people, the newspaper or a friend), internal sources (e.g., whether one said or only thought of a remark), or a combination of external and internal sources (reality monitoring; Johnson \& Raye, 1981). Of interest in the present paper is whether the generation effect often seen in item memory (better memory for internally produced items than for items presented by external sources; e.g., deWinstanley, 1995; Slamecka \& Graf, 1978) extends to source memory.

Several authors have explored generation and source memory (Brown, Jones, \& Davis, 1995; Kinjo \& Snodgrass, 2000; Rabinowitz, 1989; Riefer, Hu, \& Batchelder, 1994; Voss, Vesonder, Post, \& Ney, 1987). Of particular relevance to the present study is Jurica and Shimamura's (1999) report that whereas item recall shows the generation effect, source recognition shows a negative generation effect. Jurica and Shimamura's participants viewed three drawn faces on a computer screen, one at a time. Each face made statements (provided items) and asked participants questions to which the participants had to provide an answer (generated items). After viewing all study items, the participants spent $3 \mathrm{~min}$ recalling the topics in the study

We thank Adam R. Jones, Ginny Runyan, Audrey Swift, Jon Tetirick, and Chris Rouisse for technical assistance, and Alia Antonucci-Alter, Steve Clugston, Erik Heidefors, Tiffany Kelley, Anna Mallet, Allison Marsh, Nidhi Paul, Brett Peiffer, Lauren Seaton, Alison Stuart, Shihab Sugeir, and Courtney Ward for their help in collecting and scoring the data. Special thanks to Jason Hicks for comments on an earlier draft of this paper and to the reviewers for their thoughtful comments. This research was supported by an Abernethy Research Grant and a Faculty Study \& Research Grant, both from Davidson College, to K.D.G. and K.S.M., respectively, and is based on K.D.G.'s thesis, which was supervised by K.S.M. Portions of this research were presented at the annual meeting of the Southeastern Psychological Association in New Orleans, in March 2003, and at the Annual Meeting of the Psychonomic Society in Vancouver in November 2003. Correspondence concerning this article should be addressed to K. S. Multhaup, Box 7000, Davidson College, Davidson, NC 28035-7000 (e-mail: krmulthaup@davidson.edu). phase. Then the participants were given a source memory test. They were shown all three drawn faces and a box labeled "new topic." The test presented participants with statements and questions from the study phase and new items. For each item, participants indicated which face had used the sentence in the study phase or that the sentence was new.

A concrete example will illustrate how the Jurica and Shimamura (1999) source task fails to test generated items: Face A says "The name of Tarzan's girlfriend is Jane" (provided statement). Face B says "What is the name of the comic strip character who eats spinach to increase his strength?" (question requiring participants to generate an answer; assume the participant said "Popeye"). On the subsequent source memory test, participants are asked to identify who said "The name of Tarzan's girlfriend is Jane" (Face A provided that statement). Unfortunately, they are also asked to identify who asked "What is the name of the comic strip character who eats spinach to increase his strength?" rather than the information that was actually generated (Popeye). Given that the generation effect is defined as better memory for information that participants have produced than for information that they have been provided (Slamecka \& Graf, 1978), we argue that Jurica and Shimamura did not assess the generation effect in source memory. To do so one must ask participants about the source of what has been generated - namely, the items (e.g., Popeye; Jane would be the comparable item that was provided) rather than the questions asked and full statements made.

We argue that Jurica and Shimamura (1999) have made a valuable contribution to the literature in showing that when people are generating information they appear to reduce the encoding of the source that prompted them to generate information. These data add to the literature regarding which contextual features (e.g., repetition, source) are encoded seemingly automatically or require effort (cf. Hasher $\&$ Zacks, 1979). The data also add to what is known about the effect of generation on memory for the context in which information was presented or generated (see also Marsh, 
Edelman, \& Bower, 2001). Finally, the data demonstrate that an internal focus can reduce later memory for the source of information (see also Johnson, Nolde, \& De Leonardis [1996] and Hashtroudi, Johnson, Vnek, \& Ferguson [1994] regarding how focusing on one's emotions, another form of internal focus, later reduces memory for source). Unfortunately the data do not provide insight into the effect of generation on source memory.

In the present experiments, we explored whether a generation effect in source memory could be found with Jurica and Shimamura's (1999) procedure modified specifically to test participants on generated items. In pilot work, we simply added the participant $(I)$ to the list of possible sources and presented target words (e.g., Popeye) rather than complete statements or questions as the items for source identification. Although we did find generation effects in source memory as Brown et al. (1995) and Riefer et al. (1994) did in different paradigms, we realized that the design was biased in favor of generation effects because there was only one internal source ( $I$ generated) whereas there were three external sources (three drawn faces that provided information). To test whether generation improves source memory there must be an equal number of internal and external source options. However, if only one internal and one external source are compared, source memory is contaminated by well-documented biases such as identifying the external source for items that are familiar (the "it had to be you" bias noted by Johnson $\&$ Raye, 1981). Thus we used two internal and two external sources.

We asked participants to engage in two different tasks (unscrambling words or filling in letters to complete words) and to observe an external source completing the same tasks. This yielded two internal source options (I unscrambled the words; I filled in the letters) and two external source options (He unscrambled the words; He filled in the letters). Of interest was whether participants would be better able to discriminate internal sources as opposed to external sources - that is, whether a generation effect would be found in source memory scores.

\section{EXPERIMENT 1}

\section{Method}

Participants. Thirty Davidson College students enrolled in Psychology 101 received course credit for participating. Seventeen were female. The students' ages ranged from 18 to $22(M=19.10, S D=$ 1.03). They had an average of 12.68 years of education $(S D=0.90)$ and scored a mean of $32.93(S D=2.96)$ on the 40 -question Shipley (1940) vocabulary test.

Materials. The present study follows virtually all of Jurica and Shimamura's (1999) design. We modified their Experiment 3, which used objective trivia facts, because Slamecka and Graf (1978) argued that using "first answer that comes to mind" questions (as in Jurica \& Shimamura's [1999] Experiments 1 and 2) can yield idiosyncratic answers that bias results in favor of remembering generated items (and presumably their source). We created our own materials from the Nelson and Narens (1980) norms, which Jurica and Shimamura used to create their materials for their Experiment 3 . We randomly assigned 6 of the 30 items to be new items on the source recognition test. The remaining sentences were randomly divided into two lists: presented (statements) and generated (questions). Within these two lists, the sentences were divided into two additional levels: unscramble or fill in the letters (see below for examples). Items were counterbalanced so that every answer was completed by internal and external sources across participants. Statements and questions were presented by a face on a computer screen via a word bubble.

There were four types of items: (1) participant generated by unscrambling letters, (2) participant generated by filling in letters, (3) external source presented by unscrambling letters, and (4) external source presented by filling in letters. Whether an item was generated from a question or presented in a statement, the target answer was always the last word of the sentence. In the unscramble condition, the first two letters were moved to the end of the word (e.g., $u b$ $b e r b l)$. The fill-in-the-letters condition had all the vowels in the answer replaced by asterisks (e.g., bl*bb*r), similar to deWinstanley's (1995) task. The questions requiring generation were followed by the target answer that needed unscrambling or letters filled in (e.g., What is the name of the thick layer of fat on a whale? ubberbl What is the name of the thick layer of fat on a whale? $b l * b b * r)$. To ensure that participants did not attempt generation in presented statements (see deWinstanley, 1995), the target answer preceded the question (e.g., Blubber: The thick layer of fat on a whale is called ubberbl. Blubber: The thick layer of fat on a whale is called $b l * b b * r$.).

The face that presented the statements and asked questions requiring generation was a scanned color photograph of a middleaged, gray-haired, blue-eyed, Caucasian male $(8.5 \times 5 \mathrm{~cm})$. He lives out of state and thus was unfamiliar to the participants in this study; we obtained personal permission to use the photograph in our study.

As in Jurica and Shimamura (1999), items were presented at a 7sec rate. Presentation order was divided into six blocks of four, with each block containing two presented statements and two questions requiring generation. Items within each block were randomly ordered. The blocks were randomly assigned to an answer manipulation (fill-in-the-letters or unscramble), with the restriction that no more than two consecutive blocks could present words of the same answer manipulation.

During the source monitoring test phase, participants saw 30 words ( 24 words from study and 6 new words) presented in one of two random orders. Test words were displayed one at a time on the computer screen above five source options. The first two source options were duplicate pictures of the face, labeled He unscrambled the word and He filled in the letters, respectively. The next two options were boxes that contained the letter I in large font, labeled I unscrambled the word and I filled in the letters, respectively. The fifth option was a box that contained the letter $N$ in large font and was labeled New word.

A Dell OptiPlex Gn+ computer displayed the instructions, the study phase, and the source recognition phase. The experiment was designed with Cedrus SuperLab Pro, Windows Edition.

Procedure. The participants were tested individually; they sat in front of the computer with a researcher next to them who read aloud the study phase instructions from the computer screen. The participants were informed that for each answer, they should try to remember the source (the face or themselves) as well as which type of task the source had performed (unscrambling or filling in the letters). The participants were instructed to read all of the sentences (presented statements and questions requiring generation) silently and to answer the questions aloud (again as in Jurica \& Shimamura, 1999). An experimenter recorded the responses to the questions. Incorrect responses were infrequent (there were 9 incorrect out of a total of 360), and these were deleted from the analyses.

Following Jurica and Shimamura (1999), in the item recall phase the participants were asked to write all of the answers (final words from sentences; e.g., blubber) that they could remember, regardless of whether the answer came from a presented statement or a ques- 
tion that required generation, and regardless of the task. After 3 min, the participants were told to stop, and then they were given the source-monitoring test.

The participants read each test word and then identified the source (face unscrambled, face filled in the blank, I unscrambled, I filled in the blank) or indicated that it was a new item out loud. The experimenter recorded the responses. The task was self-paced, and once the participants had named the source, they pressed the space bar to move to the next word. The participants subsequently completed a Shipley (1940) word test.

\section{Results and Discussion}

Figure 1 shows proportion correct item recall and source recognition for target words (e.g., blubber). For item recall, participants recalled significantly more generated answers (responses to questions) $(M=0.61, S D=0.15)$ than presented answers (from statements) $(M=0.38$, $S D=0.16)[t(29)=4.16, p<.001]$. Source memory scores were calculated by dividing the number of correct source identifications (e.g., I unscrambled it, I filled in letters) by the number of correct identifications that an item was old (this is a typical source memory score, also used by Jurica \& Shimamura, 1999). Given that this scoring method excludes new items and responses, chance level performance was .25 on our task. Source recognition was above chance levels, and participants were more accurate on generated answers (responses to questions) $(M=0.73$, $S D=0.18$ ) than on presented answers (from statements) $(M=0.53, S D=0.25)[t(29)=4.07, p<.001]$.

In short, the data show a generation effect in both item and source memory. We replicated Jurica and Shimamura's (1999) generation effect in item recall, but not their negative generation effect in source recognition. Our data are, however, consistent with other findings of a genera- tion effect in source memory (see Brown et al., 1995; Riefer et al., 1994).

\section{EXPERIMENT 2}

Although Experiment 1 had experimental control (e.g., both sources did the same tasks), it is lacking in external validity for external source discrimination. In internal source discriminations in everyday settings we are sometimes called upon to determine which task we have performed (e.g., when cross-checking a table of numbers, one must remember whether rows or columns were added in order to do the complement and thus the checking). In external source discriminations in everyday settings, however, it is relatively uncommon to determine which task another person has performed. Instead, it is more common to determine which person has performed a given task. We designed Experiment 2 to have increased external validity by changing the external source from two tasks done by the same person to the same task done by one of two people. We acknowledge that some experimental control was given up in making this change. Our reasoning was that if we found the same pattern of results in Experiment 1 (high experimental control, low external validity) and Experiment 2 (less experimental control, higher external validity) then we could be assured that the data pattern was robust.

\section{Method}

Participants. Fifty-six Davidson College students participated for Psychology 101 credit. Thirty-six were female. The students' mean age was $18.23(S D=0.60)$, and they had an average of 12.11 years of education $(S D=0.41)$. Their average Shipley $(1940)$ score was 32.63 out of $40(S D=2.62)$.

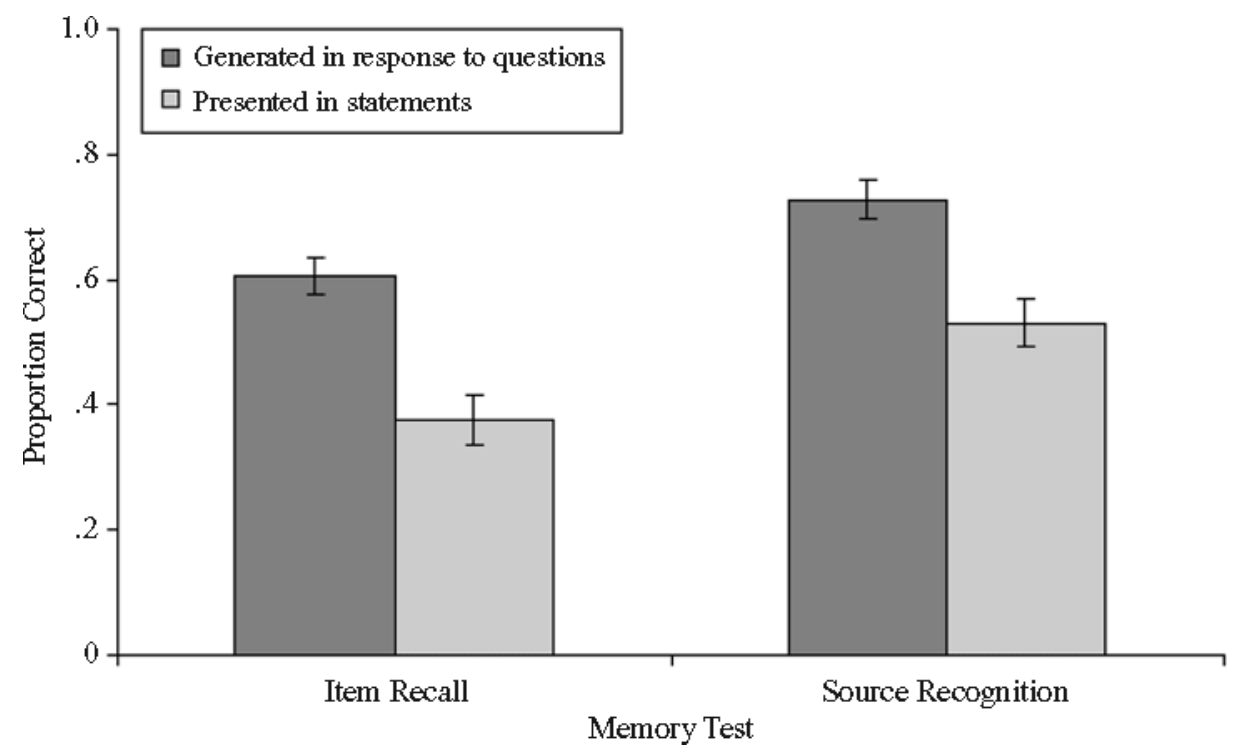

Figure 1. Mean proportion item recall and source recognition in Experiment 1; the error bars represent standard errors of the means. 
Materials. The external sources were two blonde Caucasian males who appeared to be in their late 20 s or early 30 s. They had similar features, although they were distinguishable. We obtained these pictures from two internet websites, and were granted e-mailed permission from the site owners to use the pictures.

There were four types of items: (1) participant generated by unscrambling letters, (2) participant generated by filling in letters, (3) Person A presented by making a statement, and (4) Person B presented by making a statement. The external source conditions involved one of two faces making a statement (e.g., The thick layer of fat on a whale is called blubber). The internal source conditions in which the participants unscrambled words and filled in letters were the same as in Experiment 1. Items were counterbalanced so that every answer was presented in each of the four conditions across participants. Each presentation block was randomly assigned to one external source. As in Experiment 1, there were six blocks with four sentences per block, and each block contained two statements, a question that required generation by filling in letters, and a question that required generation by unscrambling. The participants were instructed to remember which of the two external sources (faces) had given the answer for presented statements and which task they had done to generate the answer to questions.

We also manipulated the location of the external sources on the computer screen. This manipulation yielded no main effect nor was it involved in any interaction, and thus we will not discuss it further, although analyses are available upon request.

As in Experiment 1, test words were displayed one at a time on the computer screen above the five source options. The first two source options were faces, labeled Person A said the word and Person B said the word, respectively. The next two options were boxes that contained the letter $I$ in large font, labeled I unscrambled the word and I filled in the letters, respectively. The fifth option was a box that contained the letter $N$ in large font and was labeled New word.

Procedure. The procedure was the same as in Experiment 1, except that the instructions were given as a demonstration rather than an explanation. The participants saw the faces and an example item (shown as a statement and as a question, with both participant tasks demonstrated) during the instruction phase. Incorrect responses (14 out of 672 total) were deleted from the analyses.

\section{Results}

Figure 2 shows proportion correct item recall and source recognition for target words (e.g., blubber). For item recall, participants recalled significantly more generated answers (responses to questions) $(M=0.65, S D=0.14)$ than presented answers (from statements) $(M=0.31$, $S D=0.14)[t(55)=9.72, p<.001]$. Source memory scores were calculated as in Experiment 1. Again, source recognition was above chance levels $(.25)$, and participants were more accurate on generated answers (responses to questions $)(M=0.81, S D=0.14)$ than on presented answers (from statements) $(M=0.54, S D=0.27)[t(55)=$ $6.41, p<.001]$.

\section{GENERAL DISCUSSION}

Experiments 1 and 2 yield remarkably consistent findings (see Figures 1 and 2): Source recognition, like item recall, shows a generation effect (see also Brown et al., 1995; Riefer et al., 1994). Given that item memory and source memory respond similarly to generation, the most parsimonious conceptualization of memory would suggest that item and source memory do not tap distinct systems. However, there are data in the literature that suggest that in comparison with item memory, source memory can be disproportionately disrupted (e.g., Multhaup \& Balota, 1997). Thus, a more prudent view is that item memory and source memory show overlap, but not complete over-

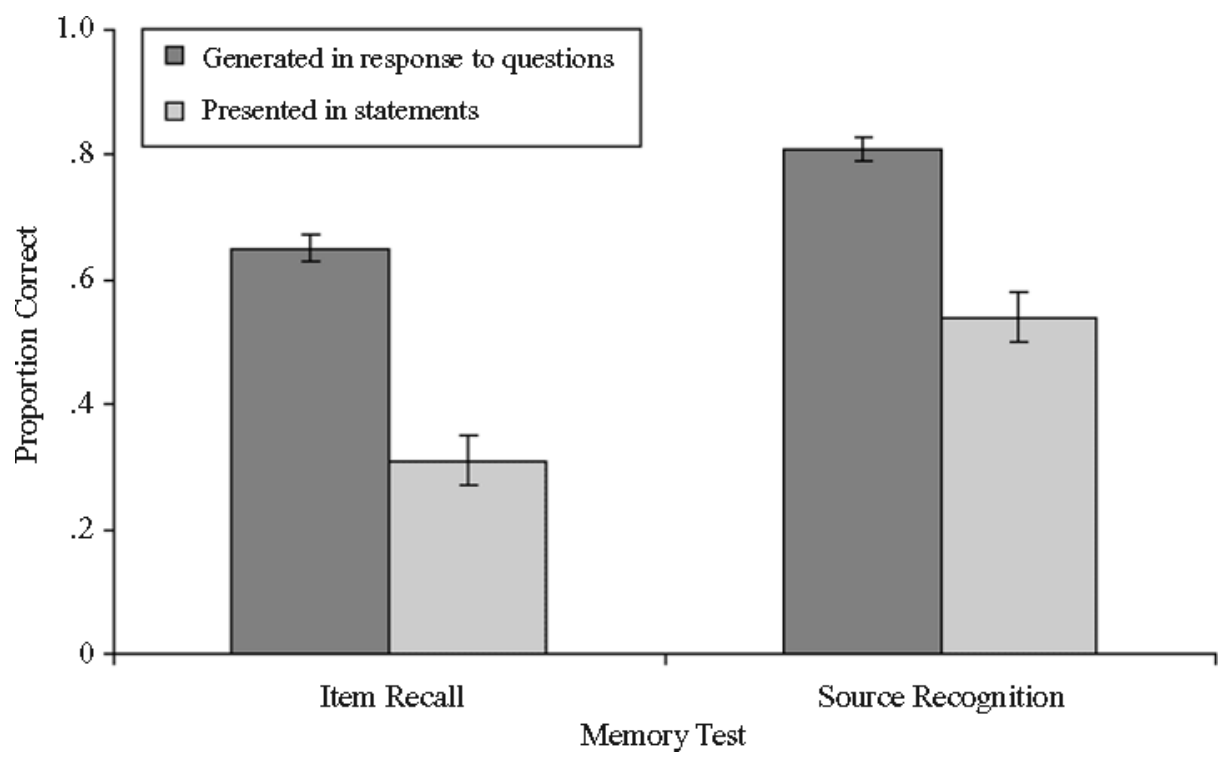

Figure 2. Mean proportion item recall and source recognition in Experiment 2; the error bars represent standard errors of the means. 
lap, in their underlying processes (see Johnson et al., 1993, for a complete discussion).

Experiment 2 can be criticized for the confound of source discrimination across external and internal sources (two people and two tasks, respectively). As we noted in the introduction to Experiment 2, our aim was to increase the external validity of our design. Thus we have consistent findings across a study with high experimental control and low external validity (Experiment 1 ) and a study with less experimental control and higher external validity (Experiment 2). In our view, the fact that the same pattern of data emerges across experiments with very different strengths and weaknesses increases the likelihood that we have detected a stable finding: Generation increases source memory performance, just as it increases item memory performance (Slamecka \& Graf, 1978).

\section{REFERENCES}

Brown, A. S., Jones, E. M., \& Davis, T. L. (1995). Age differences in conversational monitoring. Psychology \& Aging, 10, 111-122.

DeWinstanley, P. A. (1995). A generation effect can be found during naturalistic learning. Psychonomic Bulletin \& Review, 2, 538-541.

HASHER, L., \& ZACKS, R. T. (1979). Automatic and effortful processes in memory. Journal of Experimental Psychology: General, 108, 356388 .

Hashtroudi, S., Johnson, M. K., Vnek, N., \& Ferguson, S. A. (1994). Aging and the effects of affective and factual focus on source monitoring and recall. Psychology \& Aging, 9, 160-170.

Johnson, M. K., Hashtroudi, S., \& Lindsay, D. S. (1993). Source monitoring. Psychological Bulletin, 114, 3-28.

Johnson, M. K., Nolde, S. F., \& De LEONARDIS, D. M. (1996). Emo- tional focus and source monitoring. Journal of Memory \& Language, 35, 135-156.

JoHNSON, M. K., \& RAYE, C. L. (1981). Reality monitoring. Psychological Review, 88, 67-85.

JuRICA, P. J., \& ShIMAMURA, A. P. (1999). Monitoring item and source information: Evidence for a negative generation effect in source memory. Memory \& Cognition, 27, 648-656.

KinJo, H., \& SNODGRASs, J. G. (2000). Does the generation effect occur for pictures? American Journal of Psychology, 113, 95-121.

Marsh, E. J., Edelman, G., \& Bower, G. H. (2001). Demonstrations of a generation effect in context memory. Memory \& Cognition, 29, 798-805.

Multhaup, K. S., \& Balota, D. A. (1997). Generation effects and source memory in healthy older adults and in adults with dementia of the Alzheimer type. Neuropsychology, 11, 382-391.

Nelson, T. O., \& NARENS, L. (1980). Norms of 300 general-information questions: Accuracy of recall, latency of recall, and feeling-of-knowing ratings. Journal of Verbal Learning \& Verbal Behavior, 19, 338-368.

RABINOwITZ, J. C. (1989). Judgments of origin and generation effects: Comparison between young and elderly adults. Psychology \& Aging, 4, 259-268.

RiEFER, D. M., Hu, X., \& BATCHELDER, W. H. (1994). Response strategies in source monitoring. Journal of Experimental Psychology: Learning, Memory, \& Cognition, 20, 680-693.

SHIPLEY, W. C. (1940). A self-administered scale for measuring intellectual impairment and deterioration. Journal of Psychology, 9, 371377.

Slamecka, N., \& GRAF, P. (1978). The generation effect: Delineation of a phenomenon. Journal of Experimental Psychology: Human Learning \& Memory, 4, 592-604.

Voss, J. F., Vesonder, G. T., Post, T. A., \& Ney, L. G. (1987). Was the item recalled and if so by whom? Journal of Memory \& Language, 26, $\underline{466-479 .}$

(Manuscript received June 13, 2003; revision accepted for publication January 8, 2004.) 